OPEN ACCESS

Edited by:

Martin Johannes Hoogduijn,

Erasmus University

Rotterdam, Netherlands

Reviewed by:

Federica Casiraghi,

Istituto Di Ricerche Farmacologiche

Mario Negri, Italy

Evren Alici,

Karolinska Institute (KI), Sweden

Myung-Shin Jeon,

Inha University, South Korea

*Correspondence:

Marcella Franquesa

mfranquesa@igtp.cat

Specialty section:

This article was submitted to Alloimmunity and Transplantation,

a section of the journal

Frontiers in Immunology

Received: 22 January 2019

Accepted: 21 May 2019

Published: 06 June 2019

Citation:

Carreras-Planella $L$,

Monguió-Tortajada M, Borràs FE and

Franquesa M (2019)

Immunomodulatory Effect of MSC on

$B$ Cells Is Independent of Secreted

Extracellular Vesicles.

Front. Immunol. 10:1288.

doi: 10.3389/fimmu.2019.01288

\section{Immunomodulatory Effect of MSC on B Cells Is Independent of Secreted Extracellular Vesicles}

\author{
Laura Carreras-Planella ${ }^{1,2}$, Marta Monguió-Tortajada ${ }^{1,3}$, Francesc Enric Borràs ${ }^{1,2,4}$ and \\ Marcella Franquesa ${ }^{1,4 *}$ \\ ${ }^{1}$ REMAR-IVECAT Group, Germans Trias i Pujol Health Science Research Institute, Badalona, Spain, ${ }^{2}$ Department of Cell \\ Biology, Physiology and Immunology, Autonomous University of Barcelona, Barcelona, Spain, ${ }^{3}$ ICREC Research Program, \\ Germans Trias i Pujol Health Science Research Institute, Badalona, Spain, ${ }^{4}$ Nephrology Service, Germans Trias i Pujol \\ University Hospital, Badalona, Spain
}

Mesenchymal stem or stromal cells (MSC) have proven immunomodulatory properties toward B cell activation and induce regulatory B cells (Breg), through a dual mechanism of action that relies both on cell contact and secreted factors. One of them are MSC-derived extracellular vesicles (EVs), membrane nanovesicles that mediate cell communication and typically reflect the phenotype of the cell of origin. MSC-EVs could resemble MSC functions, and are being contemplated as an improved alternative to the MSC-based immunomodulatory therapy. In the present work, we focused on the factors secreted by MSC and aimed to elucidate the putative role of MSC-EVs in the immunomodulation of B cells. EVs and soluble protein-enriched fractions (PF) were isolated from MSC-conditioned medium (CM) using size-exclusion chromatography (SEC) and their capacity to modulate B cell activation, induction of Breg and B cell proliferation was compared to that of the whole MSCs. Co-culture with MSC or unfractionated CM induced naïve and CD24 ${ }^{\text {hi }} \mathrm{CD} 38^{\text {hi }}$, IL-10 producing (Breg) phenotypes on B cells while not affecting proliferation. MSC-PF had a comparable effect to MSCs, inducing a naivve phenotype, and even though they did not induce the shift toward a CD24 ${ }^{\text {hi }}$ CD38 ${ }^{\text {hi }}$ population, MSC-PF fostered IL-10 production by B cells. Conversely, MSC-EVs failed to promote naïve B cells and to reduce memory B cells. MSC-EVs induced CD24 ${ }^{\text {hi }} \mathrm{CD} 38^{\text {hi }} \mathrm{B}$ cells to a similar extent of that of MSC, but not bona fide Bregs since they did not produce IL-10. Our results show that B cell modulation by MSC is partially mediated by soluble factors other than EVs.

Keywords: mesenchymal stromal cells, exosome, regulatory B cell, immunosuppression, memory B cell, Ev isolation

\section{INTRODUCTION}

Mesenchymal stem or stromal (MSC) are immunomodulatory toward numerous immune cell types in vitro as well as in vivo (1-3). We recently showed their ability to induce regulatory (Breg) and naïve $B$ cells while reducing activated and memory B cells (4). While the exact mechanism of action remains unclear (5), both cell-contact and secreted factors are needed for MSC modulation of B cells $(6,7)$. Some cytokines and growth factors have been identified as key mediators amid secreted 
factors, but more recently the focus has been put on extracellular vesicles (EVs). EVs are membrane nanovesicles that carry molecules reflecting the phenotype and functions of the cells of origin (8). MSC-derived EVs have been shown to emulate their effect on $\mathrm{B}$ cells and other immune cells (9-11). However, parameters related to the EV isolation method -including purity- are key to downstream analyses. Widely used techniques such as ultracentrifugation (UC) or precipitating agents-based methods cause the co-precipitation of EVs with other potentially confusing soluble molecules (12), whilst sizeexclusion chromatography (SEC) is being considered the method of choice to highly enrich functional EVs (13).

The purpose of the present study is to use SEC to dissect the role of MSC-EV from secreted soluble factors in order to deepen in the mechanisms of B cell immunomodulation by MSC.

\section{MATERIALS AND METHODS}

\section{Mesenchymal Stem or Stromal Cell Isolation and Cell Culture}

Subcutaneous adipose tissue was obtained from patients undergoing heart surgery in University Hospital Germans Trias i Pujol (HUGTiP). Informed consent was obtained from all subjects, and the study protocol conformed to the principles outlined in the Declaration of Helsinki. Mesenchymal stem or stromal cells (MSC) were isolated from fat tissue as previously described $(4,14)$. MSC, which were used in passages between 3 and 10 , were cultured in $\alpha$ MEM (Sigma Aldrich) supplemented with 10\% FBS (Lonza), penicillin (100 IU/ml, Cepa S.L., Madrid, Spain), streptomycin (100 mg/ml, Normon Laboratories S.A., Madrid, Spain) and $2 \mathrm{mM}$ L-Glutamine (Sigma Aldrich).

\section{Preparation of Conditioned Medium}

Two million MSC were seeded in cell culture flasks with $15 \mathrm{ml}$ of complete medium depleted from fetal bovine serum (FBS)derived EVs (11). To deplete medium from FBS-EVs, 20\% FBS complete medium $(\alpha \mathrm{MEM}+1 \% \mathrm{P} / \mathrm{S}+2 \mathrm{mM}$ L-Glutamine $)$ was ultracentrifuged at $100,000 \times g$ for $16 \mathrm{~h}$ in polypropylene ultracentrifugation tubes (Beckman coulter, Brea, CA). The supernatant was collected and filtered through a $0.22 \mu \mathrm{m}$ filter (Sarstedt, Germany) to sterilize the medium, which was finally diluted with $\alpha$ MEM medium to the final concentration of $10 \%$ FBS for cell culture. After $48 \mathrm{~h}$, the medium was collected and centrifuged at $400 \times g$ and $2,000 \times g$ to eliminate cells and cell debris, respectively, to obtain MSC-conditioned medium (CM).

\section{Extracellular Vesicles and Soluble Protein Separation and Analysis Size-Exclusion Chromatography}

MSC-CM was concentrated using a $100 \mathrm{kDa}$ ultrafiltration unit (Amicon Ultra, Millipore, Millerica MA) and fractioned by SEC using columns of $1 \mathrm{ml}$ sepharose CL-2B (Sigma Aldrich). Figure 1A schematically depicts the followed protocol, which can be read in detail in Monguió-Tortajada et al. (15).

\section{Bead-Based Flow Cytometry}

The EV-enriched SEC fractions were determined by beadsbased flow cytometry according to the presence of CD9 and CD90 following the previously described procedure $(11,15)$ (Figure 1B). Briefly, EVs were coupled to $4 \mu \mathrm{m}$ aldehyde/sulfate latex microspheres and were then labeled with the fluorochromeconjugated antibodies anti-CD90-PE-Cy7 or indirectly labeled with the primary antibodies anti-CD9 (Clone VJ1/20) and or the IgG isotype control and secondary antibody FITC-conjugated Goat F(ab')2 Anti-Mouse IgG. The MSC-EV fractions with the highest CD9 and CD90 MFI were pooled to obtain highly enriched MSC-EV preparations.

\section{Cryo-Transmission Electron Microscopy (Cryo-TEM)}

The presence of EVs in EV-enriched fractions was confirmed by cryo-transmission electron microscopy (cryo-TEM) as previously described (16).

\section{Protein Quantification}

Protein elution was checked by reading the absorbance (Abs.) at $280 \mathrm{~nm}$ of each fraction using Nanodrop ${ }^{\circledR}$ ND-1000 (Thermo Scientific) to pool fractions with the highest protein concentration and obtain MSC-PF preparations.

\section{B Cells}

\section{Isolation From Tonsils}

Tonsils were obtained from children undergoing routine tonsillectomy after the informed consent of their legal tutors (HUGTiP). The study protocol followed the principles of the Declaration of Helsinki. To obtain B cells, tonsils were mechanically disaggregated with a scalpel, washed with PBS, mononuclear cells were isolated by ficoll differential centrifugation (GE Healthcare) and frozen (liquid $\mathrm{N}_{2}$ ). For the experiments, tonsil cells were thawed and negatively sorted (MACS, Miltenyi Biotech) to obtain the $\mathrm{CD}^{-} 3^{-}$population (mature inactivated B cells).

\section{Activation and Culture}

One hundred-thousand B cells were seeded in flat bottom 96 well plates, T-cell-like-stimulated with IL-2/anti-IgM/anti-CD40 and co-cultured with 10,000 MSC for 7 days in a 10:1 B:MSC ratio as previously described $(4,7)$.

B cells were also cultured with MSC-CM, MSC-EV, or MSCPF. Of each condition, $100 \mu \mathrm{l}$ of MSC-EV or MSC-PF were added to the culture. This volume corresponded to EVs and PF secreted by 50,000 MSC which is 5 times the amount of MSC added in the B cell-MSC co-culture. In the dose-dependency experiments, half $(50 \mu \mathrm{l})$ or one tenth $(10 \mu \mathrm{l})$ of the initial volume of $\mathrm{CM}$ or PF conditions were used.

\section{B Cell Subsets and Proliferation Assessment by Flow Cytometry}

After 7 days, B cells were collected and processed for flow cytometric analysis to analyze B cell populations (FACS Canto II, BD Biosciences) as previously described (4, 7). Viability was assessed by 7AAD staining (BD Biosciences) and quantified as $\mathrm{CD}_{1}{ }^{+} 7 \mathrm{AAD}^{-}$cells among the lymphocyte population. In separate experiments, B cells were labeled with 


\section{A}
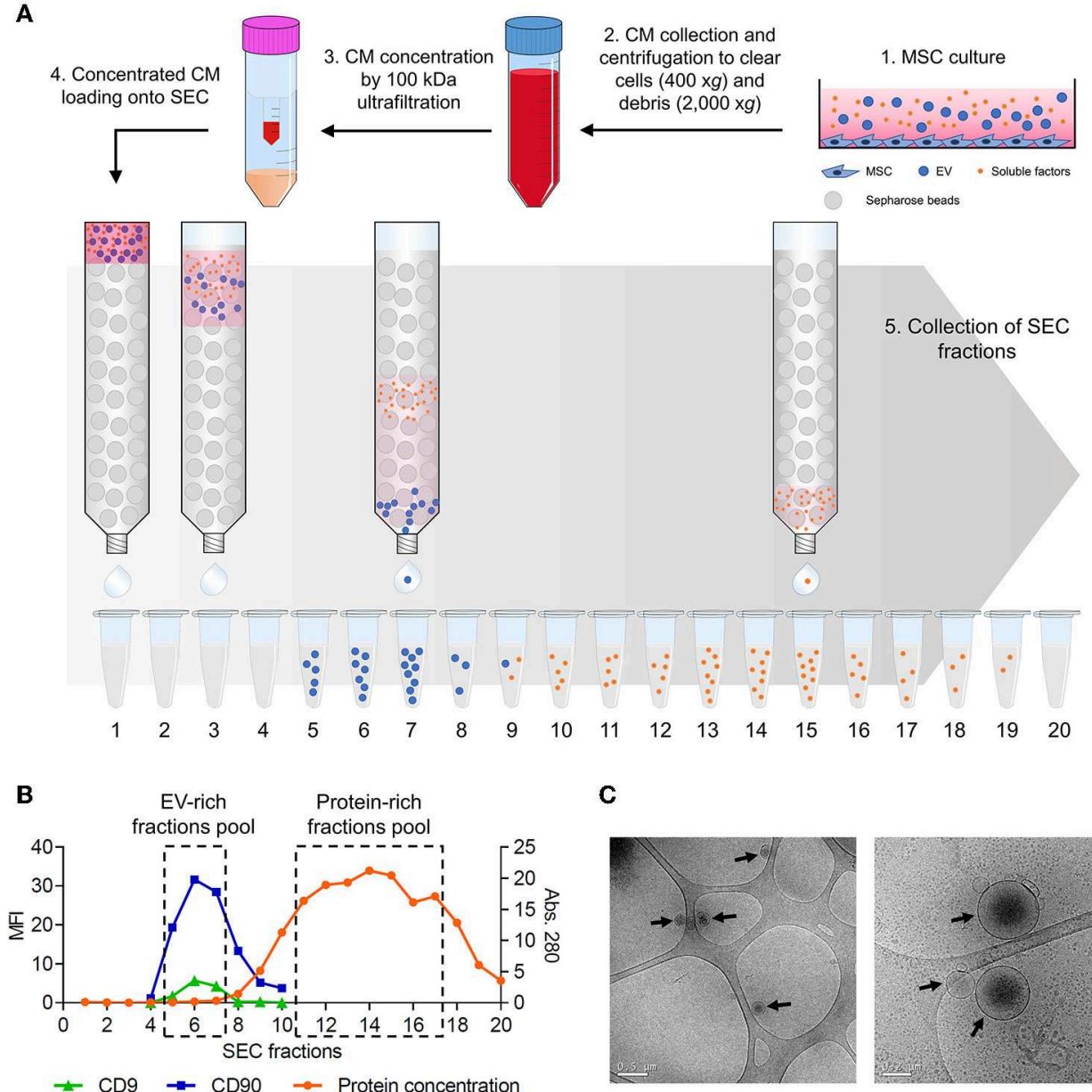

C
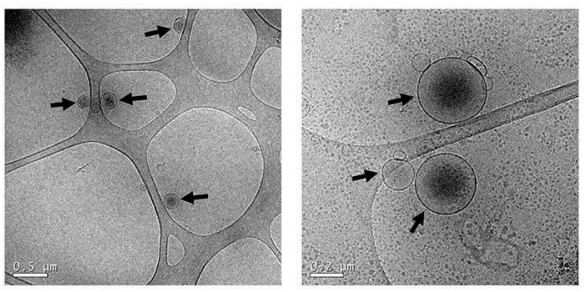

D
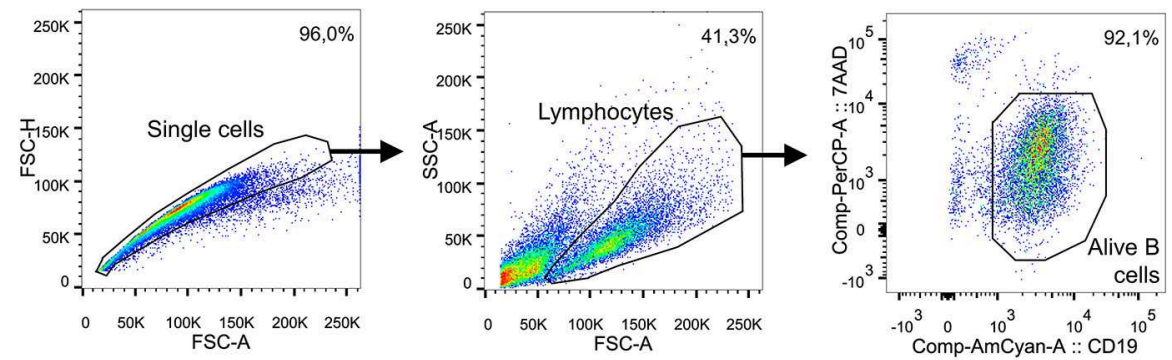

From alive $B$ cells:
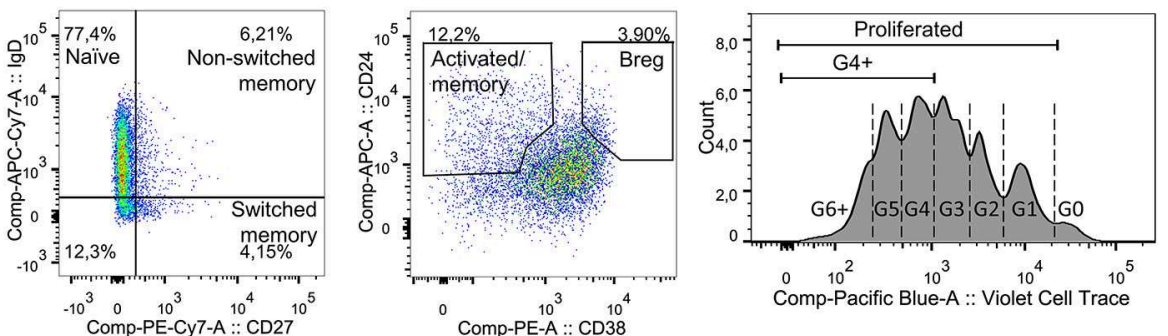

FIGURE 1 | (A) Workflow of the methodology used to isolate MSC-EV and MSC-PF by SEC from MSC-CM. (1) Supernatant was collected after $48 \mathrm{~h}$ of MSC culture and (2) sequentially centrifuged at $400 \times g$ for $5 \mathrm{~min}$ and at 2,000 $\times g$ for $10 \mathrm{~min}$ to exclude cells and cell debris, respectively. The obtained MSC-CM was partially kept for experimental use in B cell culture and the rest was then (3) concentrated by $100 \mathrm{kDa}$ ultrafiltration. (4) Concentrated MSC-CM was loaded into a $1 \mathrm{ml}$ SEC 
FIGURE 1 | sepharose column and (5) $100 \mu$ f fractions (up to 20) were collected immediately after loading. (B) Representative plot showing the SEC elution profile according to protein concentration and median fluorescence intensity (MFI) of EV markers of each SEC fraction. Fractions with the highest CD9 and CD90 MFI or were pooled together, similarly to fractions with the highest protein concentration, to obtain the MSC-EV or MSC-PF preparations, respectively. (C) Cryo-TEM was used to analyze MSC-EV size and morphology. Black arrows point EV in the preparations over the TEM grid. Scale bars $=0.5 \mu \mathrm{m}$ (left) and $0.2 \mu \mathrm{m}$ (right). (D) Gating strategy followed to define B cell subsets by flow cytometry. Doublets and debris were excluded by FSC-A/FSC-H and FSC-A/SSC-A, respectively, by gating on singlets and lymphocyte populations. Alive $B$ cells were further gated as $C D 19^{+} 7 \mathrm{AAD}^{-}$, from which each subset was defined: naïve $\left(\mathrm{CD}^{2} 7^{-} \lg \mathrm{D}^{+}\right)$, non-switched memory $\left(\mathrm{CD} 27^{+} \operatorname{lgD}{ }^{+}\right)$, switched memory $\left(\mathrm{CD} 27^{+} \operatorname{lgD}{ }^{-}\right)$, activated/memory $\left(\mathrm{CD} 24^{\mathrm{hi}} \mathrm{CD} 38^{\mathrm{int} / \mathrm{lo}}\right)$, and Breg $\left(\mathrm{CD} 24^{\mathrm{hi}} \mathrm{CD} 38^{\mathrm{hi}}\right)$. Bottom right panel shows a representative proliferation plot where cell generations are indicated with dashed lines. For analysis, the percentages of total proliferated cells (G1 onwards) and G4+ proliferated cells (G4 onwards) were calculated.

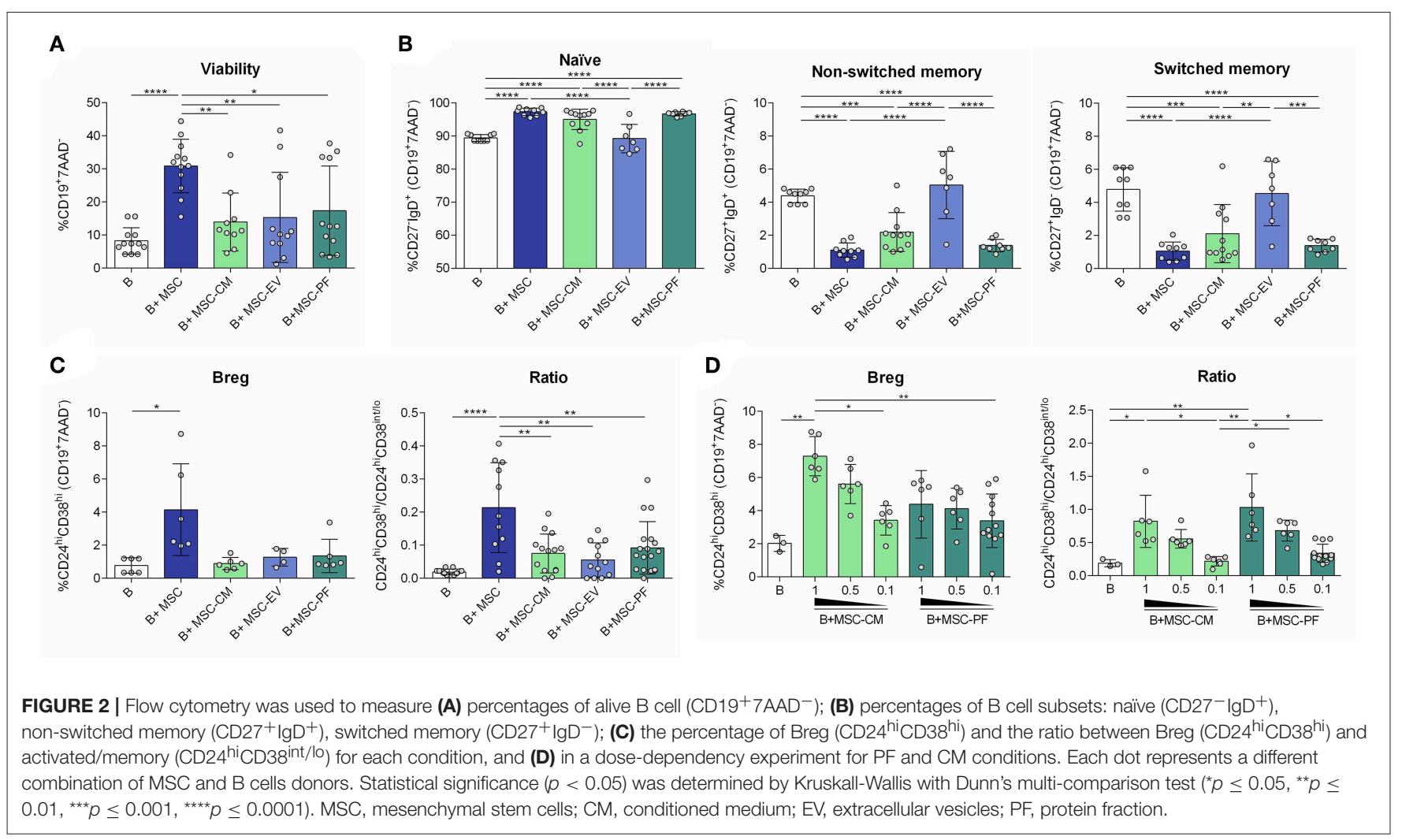

Violet CellTrace (Invitrogen) for $20 \mathrm{~min}$ at $37^{\circ} \mathrm{C}$ to analyze proliferation by dye dilution. Figure 1D shows the gating strategy followed.

\section{IL-10 and TNF $\alpha$ Quantification by ELISA}

After co-culture, supernatants were harvested and stored at $-80^{\circ} \mathrm{C}$ for later IL- 10 and TNF $\alpha$ quantification using dedicated ELISA kits (U-CyTech).

\section{Data Analysis}

All the experiments were performed with an allogenic co-culture of MSC and B cells from eleven and five different donors, respectively. Technical duplicates were used. Flow cytometry data was analyzed by FlowJo v10 software (FlowJo LLC, Ashland, OR). Statistical analysis was performed with Prism v6 software (GraphPad, La Jolla, CA). Kruskall-Wallis with Dunn's multicomparison test was used to determine statistical differences between groups ( $p<0.05$ was considered significant).

\section{RESULTS}

\section{EV Isolation and Analysis}

MSC-CM obtained after $48 \mathrm{~h}$ of culture was collected concentrated and fractionated by SEC. Fractions eluted from SEC were evaluated for CD9 and CD90, which are known EV (8) and MSC-EV markers (17), respectively (Figure 1A). As previously described by our group (11), we consistently observed that SEC EV-enriched fractions were free from the bulk of soluble proteins, that eluted in later fractions. MSC-EV were further characterized by Cryo-TEM, confirming the presence of nanosized EV (Figure 1C).

\section{B Cell Survival Relies on Cell-To-Cell Contact}

We have previously shown that MSC increase B cell survival compared to non-stimulated $\mathrm{B}$ cells but also compared to $\mathrm{T}$ cell-like activated B cells (IL-2/anti-IgM/anti-CD40) (4). In the current setting, the number of living $\mathrm{B}$ cells $\left(\mathrm{CD} 19^{+} 7 \mathrm{AAD}^{-}\right)$ 
was significantly increased in the co-cultures with MSC but not in the other conditions, compared to stimulated B cells alone (Figure 2A).

\section{B Cell Plasticity Mediated by MSC Is Independent of MSC-EVs}

$B$ cell population analysis by flow cytometry revealed that MSC$\mathrm{CM}$ and MSC-PF retained B cells in a naïve state similarly to MSC contact, and reduced non-switched and switched memory B cells. MSC-EV did not change B cell populations of stimulated B cells (Figure 2B).

\section{The MSC-PF but Not MSC-EV Is Partially Responsible of Breg Induction}

We next assessed the induction of Breg, phenotypically defined here as $\mathrm{CD} 24^{\mathrm{hi}} \mathrm{CD} 38^{\mathrm{hi}} \mathrm{CD} 19^{+} \mathrm{B}$ cells and by secretion of IL-10 and low secretion of $\mathrm{TNF} \alpha$. The $\mathrm{CD} 24^{\mathrm{hi}} \mathrm{CD} 38^{\text {hi }}$ phenotype was only significantly induced by MSC co-culture. Since activated and memory $\mathrm{B}$ cells represent the $\mathrm{CD} 24^{\text {hi }} \mathrm{CD} 38^{\text {int }} / \mathrm{lo}$ subset, we defined the ratio $\mathrm{CD} 24^{\mathrm{hi}} \mathrm{CD} 38^{\mathrm{hi}} / \mathrm{CD} 24^{\mathrm{hi}} \mathrm{CD} 38^{\text {int }} / \mathrm{lo}$ as an index between transitional immunosuppressive $\left(\mathrm{CD} 24^{\mathrm{hi}} \mathrm{CD} 38^{\mathrm{hi}}\right)$ and inflammatory activated/memory $\left(\mathrm{CD} 24^{\text {hi }} \mathrm{CD} 38^{\text {int } / l o}\right) \quad B$ cell populations. In this case, MSC significantly generated a higher immunoregulatory ratio than any of the other conditions (Figure 2C).

To further confirm that the paracrine effect of MSC on Breg polarization is mediated by the PF of the CM, a dose-dependency experiment was performed. We observed that the percentage of $\mathrm{CD} 24^{\mathrm{hi}} \mathrm{CD} 38^{\mathrm{hi}}$ cells and the ratio $\mathrm{CD} 24^{\text {hi }} \mathrm{CD} 38^{\mathrm{hi}} / \mathrm{CD} 24^{\mathrm{hi}} \mathrm{CD} 38^{\text {int }} / \mathrm{lo}$ were higher when the highest dose of PF and CM was added to cells, showing that this effect is related to the $\mathrm{CM}$ and its soluble protein-enriched fractions (Figure 2D). Moreover, the PF as well as the CM could increase the percentage of non-switched and switched memory subsets in a dose-dependent manner (data not shown).

In line, the IL-10 concentration of MSC co-culture condition was also significantly higher than in any other condition, except for MSC-PF. MSC-PF significantly induced more IL10 secretion than MSC-EV and B cells alone. Noticeably, $\mathrm{TNF} \alpha$ was secreted at very low concentrations in all conditions $(<20 \mathrm{pg} / \mathrm{ml})$ (Figure 3).

\section{Assessment of B Cell Subsets Proliferation}

We also compared the effect of whole MSC with their secreted factors in terms of B cell subsets proliferation. In our setting, after 7 days $>80 \%$ of $\mathrm{B}$ cells proliferated in all conditions with few changes between groups (data not shown), so we focused on the study of 4th generation onwards proliferation $(\mathrm{G} 4+)$. MSC co-culture allowed B cell proliferation G4 onwards whilst MSC-CM and its derived fractions did not reach the same percentage, even though the differences did not reach statistical significance. We further observed a trend of MSC-EV compared MSC-PF to reduce proliferation of naïve, non-switched memory B cells and Bregs while increasing switched memory proliferation (Figure 4).

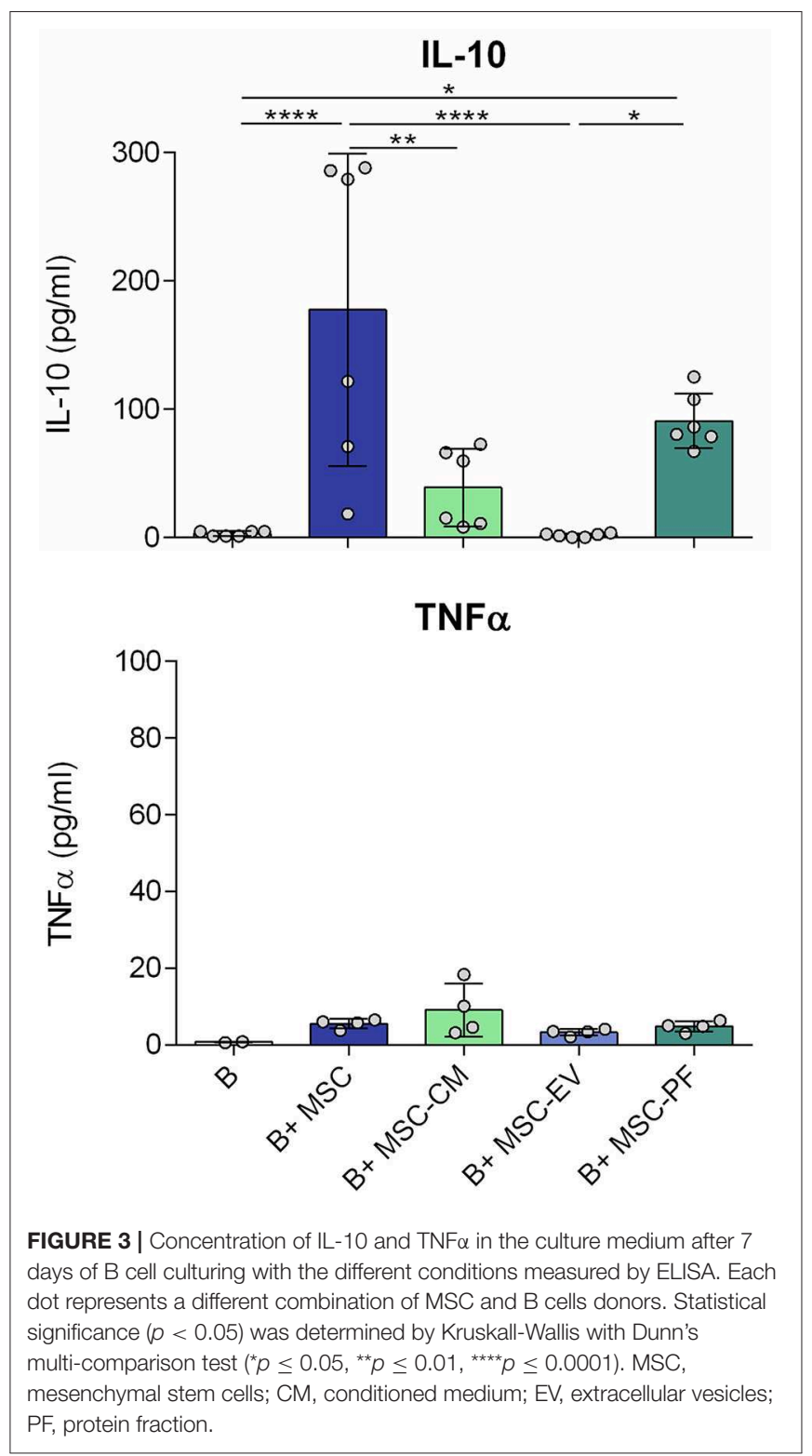

\section{DISCUSSION}

In the present work we studied the role of the MSC secreted factors on B cell immunomodulation. We isolated EVs by SEC, which allows to obtain purer EV preparation to properly discriminate it from the non-EV soluble fraction.

Ultracentrifugation (UC) without any extra purification step has been the solely and most widely used method for EV isolation, as reflected in the majority of published articles. However, UC is not an appropriate method to isolate EV since it affects the biophysical properties and co-precipitate EV with other particles (proteins, membrane fractions...) that are interfering with the subsequent molecular or functional analyses (18-20). Recently, more refined techniques for EV purification have made their way into the field which are slowly 

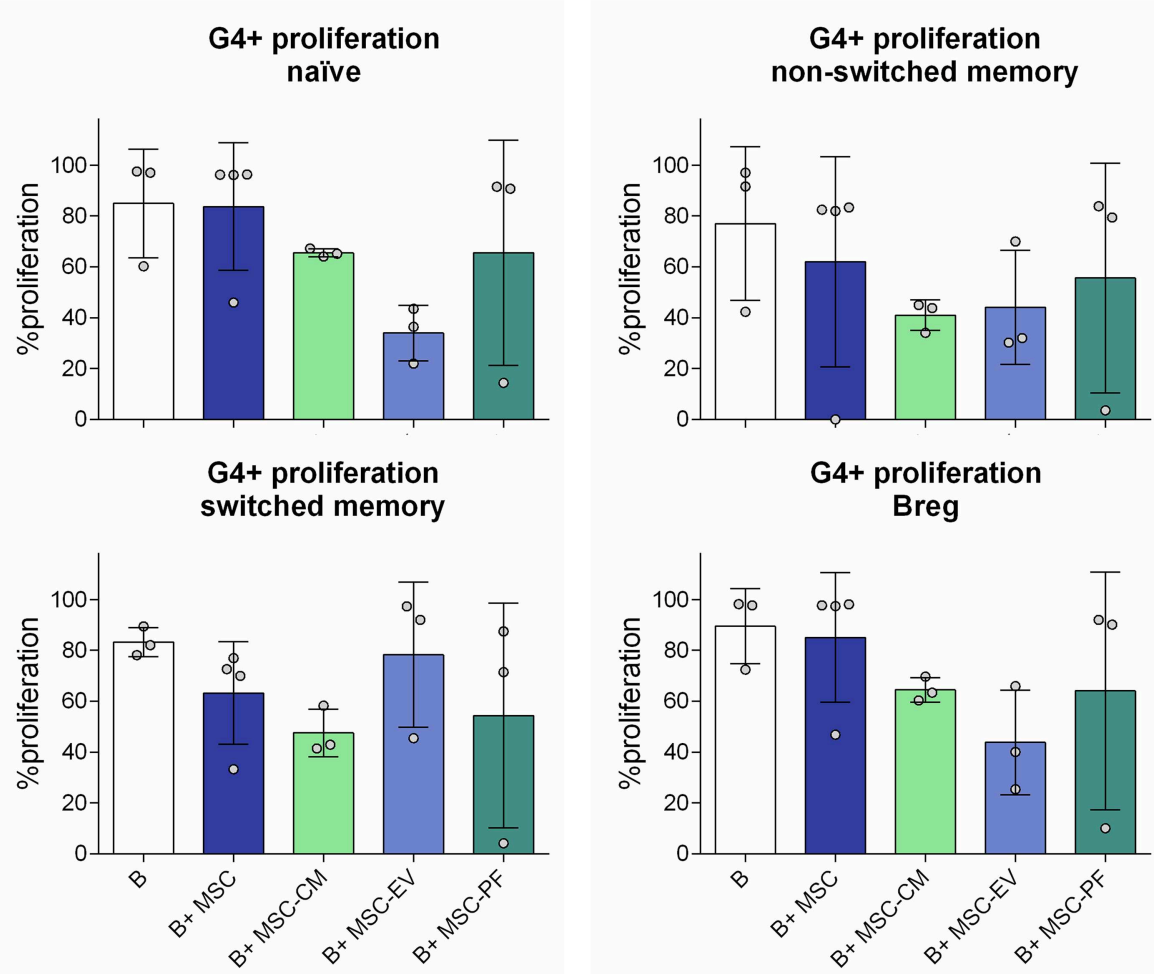

FIGURE 4 | Percentage of proliferation from the 4th generation onwards (G4+ proliferation) of the different B cell subsets measured by dilution of Violet CellTrace dye by flow cytometry. The different subsets were gated from alive B cells (CD19+7AAD ${ }^{-}$). Naïve, CD19 ${ }^{+} \mathrm{CD}^{+} 7^{-}$IgD ${ }^{+}$; non-switched memory, CD27+ IgD+ ; switched memory, CD27+ ${ }^{+} \mathrm{gD}^{-}$; Breg, CD24 ${ }^{\text {hi }} \mathrm{CD} 38^{\text {hi }}$. No statistically significant differences were found between conditions by Kruskall-Wallis with Dunn's

multi-comparison test.

changing the paradigm about the properties and functional characteristics of EV. Within these methods, SEC stands out for its better preservation, higher yield and purity, and ease for EV isolation $(12,21)$. Several groups have assessed the differences between UC and SEC for downstream analyses, concluding that UC co-precipitate EVs and soluble proteins hampering the discrimination of their individual effect in functional studies $(11,13,22,23)$.

Conditioned medium (MSC-CM) was fractionated by SEC to obtain EV-enriched and non-EV soluble protein fractions, and their distinct effect on $\mathrm{B}$ cell subsets and proliferation after Tcell like activation was analyzed. MSC-CM and MSC-PF had a comparable immunomodulatory effect to that of MSC on B cells-increasing the proportion of naïve B cells and IL-10 production and reducing memory B cell phenotype proportions. In contrast, MSC-EV did not exert any effect on activated B cells. Our results contrast with those obtained before that postulate MSC-EV as important mediators for B cell immunomodulation $(9,24)$. One of the key points of this difference is the method used to isolate and purify the EVs.

The effect of MSC-PF is similar but to a lower level than MSC co-culture. This can be due to the fact that the modulation of B cells by MSC is partially cell-contact dependent, but it also could be explained by differences in B cells' stimulation time frame, since factors are secreted continuously throughout 7 days in the co-culture. To counterbalance this effect, and because a well-defined quantification of EVs is missing in the field, the amount of MSC-EV and MSC-PF used was coming from a proportionally higher number of cells than those used in the co-culture setting. This approach avoids the potential underestimation of the effect of EV or soluble factors due to an insufficient amount.

The lack of unique markers for Breg complicates their analysis. While the transitional phenotype $\mathrm{CD} 24^{\text {hi }} \mathrm{CD} 38^{\text {hi }}$ appears as one of the most accepted, IL-10 production is still the key feature to define Breg. In our hands, the transitional phenotype did not fully correlate with IL-10 secretion, since the percentage of $\mathrm{CD} 24{ }^{\text {hi }} \mathrm{CD} 38^{\text {hi }}$ was only increased in the MSC co-culture setting compared to $\mathrm{B}$ cells alone, while IL-10 concentration was significantly increased in both the co-culture as well as with MSC-PF. However, we experimentally observed that the ratio transitional $\left(\mathrm{CD} 24^{\text {hi }} \mathrm{CD} 38^{\text {hi }}\right) /$ activated-memory B cells (CD24 ${ }^{\text {hi }} \mathrm{CD} 38^{\text {int } / \mathrm{lo}}$ ) correlated better with IL-10 production. This argues for the hypothesis that a favorable balance between immunomodulatory and pro-inflammatory phenotypes is needed for immunomodulation, rather than fostering immunomodulatory phenotypes alone. 
Proliferation experiments rendered a similar percentage of proliferating cells in B cells alone and MSC conditions. However, as the viability of B cells alone was significantly lower, the total number of proliferated B cells was higher in the MSC co-culture. Results were not significantly different between MSC-CM or its derived fractions, but the mean proliferation percentage was lower than in the co-culture, suggesting a cell-contact based mechanism to fulfill an optimal MSC conditioning. Still, we observed a trend of MSC-CM and MSC-PF to better reflect the effect of MSC co-culture compared to MSC-EV supporting the idea that MSC-PF better reflects the immunomodulatory effect of MSC.

Using SEC as one of the most refined current techniques to separate CM's EV and soluble proteins, we can conclude that the partial effect of MSC soluble factors on B cell immunomodulation is preferentially induced not by EV but rather by the proteinenriched fractions. The actual factors responsible of the effect are still under investigation.

\section{ETHICS STATEMENT}

This study was carried out in accordance with the recommendations of Guideline for Good Clinical Practice from the Comitè d'Ética de la investigació clínica de l'Hospital Universitari Germans Trias i Pujol with written informed consent from all subjects. All subjects gave written informed consent in accordance with the Declaration of Helsinki. The protocol was approved by the Comitè d'Etica de

\section{REFERENCES}

1. Hoogduijn MJ, Popp F, Verbeek R, Masoodi M, Nicolaou A, Baan C, et al. The immunomodulatory properties of mesenchymal stem cells and their use for immunotherapy. Int Immunopharmacol. (2010) 10:1496-500. doi: 10.1016/j.intimp.2010.06.019

2. Le Blanc K, Mougiakakos D. Multipotent mesenchymal stromal cells and the innate immune system. Nat Rev Immunol. (2012) 12:383-96. doi: $10.1038 /$ nri3209

3. Gao F, Chiu SM, Motan DAL, Zhang Z, Chen L, Ji H-L, et al. Mesenchymal stem cells and immunomodulation: current status and future prospects. Cell Death Dis. (2016) 7:e2062. doi: 10.1038/cddis.2015.327

4. Franquesa M, Mensah FK, Huizinga R, Strini T, Boon L, Lombardo E, et al. Human adipose tissue-derived mesenchymal stem cells abrogate plasmablast formation and induce regulatory B cells independently of T helper cells. Stem Cells. (2015) 33:880-91. doi: 10.1002/stem.1881

5. Franquesa M, Hoogduijn MJ, Bestard O, Grinyó JM. Immunomodulatory effect of mesenchymal stem cells on B cells. Front Immunol. (2012) 3:212. doi: 10.3389/fimmu.2012.00212

6. Luk F, de Witte SFH, Korevaar SS, Roemeling-van Rhijn M, Franquesa $\mathrm{M}$, Strini $\mathrm{T}$, et al. Inactivated mesenchymal stem cells maintain immunomodulatory capacity. Stem Cells Dev. (2016) 25:1342-54. doi: $10.1089 / \mathrm{scd} .2016 .0068$

7. Luk F, Carreras-Planella L, Korevaar SS, de Witte SFH, Borràs FE, Betjes $\mathrm{MGH}$, et al. Inflammatory conditions dictate the effect of mesenchymal stem or stromal cells on B cell function. Front Immunol. (2017) 8:1042. doi: 10.3389/fimmu.2017.01042

8. Yáñez-Mó M, Siljander PRM, Andreu Z, Bedina Zavec A, Borràs FE, Buzas EI, et al. Biological properties of extracellular vesicles and their physiological la investigació clínica de l'Hospital Universitari Germans Trias i Pujol.

\section{AUTHOR CONTRIBUTIONS}

LC-P contributed to collection of data analysis and interpretation and manuscript writing. MM-T and FB contributed to data analysis and interpretation, and final approval of manuscript. MF conception and design, collection of data, data analysis and interpretation, and manuscript writing.

\section{ACKNOWLEDGMENTS}

This work was supported in part by Fundació La Marató de TV3 (201516-10, 201502-30), SGR programme of Generalitat de Catalunya (2017-SGR-301 REMAR Group), ISCIII-REDinREN (RD16/0009 Feder Funds) and Instituto Carlos III project PI17/00335, integrated in the National R+ D+ I and funded by the ISCIII and the European Regional Development Fund. LC-P by the Spanish Government FPU grant (Formación de Personal Universitario, FPU17/01444); MM-T is sponsored by the PERIS (SLT002/16/00234) from the Generalitat de Catalunya; FB is a researcher from Fundació Institut de Recerca en Ciències de la Salut Germans Trias i Pujol, supported by the Health Department of the Catalan Government (Direcció General de Recerca i Innovació, Department Salut, Generalitat de Catalunya) and MF is funded by the Catalan Health Department (Generalitat de Catalunya) contract PERIS (SLT002/16/00069); the authors also want to thank Miriam Morón-Font for the graphical art help.

functions. J Extracell Vesicles. (2015) 4:27066. doi: 10.3402/jev.v4. 27066

9. Budoni M, Fierabracci A, Luciano R, Petrini S, DiCiommo V, Muraca M. The immunosuppressive effect of mesenchymal stromal cells on b lymphocytes is mediated by membrane vesicles. Cell Transplant. (2012) 22:369-79. doi: 10.3727/096368912X653309

10. Di Trapani M, Bassi G, Midolo M, Gatti A, Takam Kamga P, Cassaro A, et al. Differential and transferable modulatory effects of mesenchymal stromal cellderived extracellular vesicles on T, B and NK cell functions. Sci Rep. (2016) 6:24120. doi: 10.1038/srep24120

11. Monguió-Tortajada M, Roura S, Gálvez-Montón C, Pujal JM, Aran G, Sanjurjo L, et al. Nanosized UCMSC-derived extracellular vesicles but not conditioned medium exclusively inhibit the inflammatory response of stimulated T cells: implications for nanomedicine. Theranostics. (2017) 7:270-84. doi: 10.7150/thno.16154

12. Gámez-Valero A, Monguió-Tortajada M, Carreras-Planella L, Franquesa $\mathrm{M}$, Beyer $\mathrm{K}$, Borràs FE. Size-exclusion chromatography-based isolation minimally alters extracellular vesicles' characteristics compared to precipitating agents. Sci Rep. (2016) 6:33641. doi: 10.1038/srep 33641

13. Mol EA, Goumans M-J, Doevendans PA, Sluijter JPG, Vader P. Higher functionality of extracellular vesicles isolated using size-exclusion chromatography compared to ultracentrifugation. Nanomed Nanotechnol Biol Med. (2017) 13:2061-5. doi: 10.1016/j.nano.2017.03.011

14. Perea-Gil I, Prat-Vidal C, Bayes-Genis A. In vivo experience with natural scaffolds for myocardial infarction: the times they are a-changin'. Stem Cell Res Ther. (2015) 6:248. doi: 10.1186/s13287-015-0237-4

15. Monguió-Tortajada M, Morón-Font M, Gámez-Valero A, Carreras-Planella L, Borràs FE, Franquesa M. Extracellular-vesicle isolation from different 
biological fluids by size-exclusion chromatography. Curr Protoc Stem Cell Biol. (2019) 30:e82. doi: 10.1002/cpsc.82.

16. Lozano-Ramos I, Bancu I, Oliveira-Tercero A, Armengol MP, Menezes-Neto A, Portillo HAD, et al. Size-exclusion chromatography-based enrichment of extracellular vesicles from urine samples. J Extracell Vesicles. (2015) 4:27369. doi: $10.3402 /$ jev.v4.27369

17. van Balkom BWM, Gremmels H, Giebel B, Lim SK. Proteomic signature of mesenchymal stromal cell-derived small extracellular vesicles. Proteomics. (2019) 19:1800163. doi: 10.1002/pmic.201800163

18. Baranyai T, Herczeg K, Onódi Z, Voszka I, Módos K, Marton N, et al. Isolation of exosomes from blood plasma: qualitative and quantitative comparison of ultracentrifugation and size exclusion chromatography methods. PLoS ONE. (2015) 10:e0145686. doi: 10.1371/ journal.pone. 0145686

19. Linares R, Tan S, Gounou C, Arraud N, Brisson AR. High-speed centrifugation induces aggregation of extracellular vesicles. J Extracell Vesicles. (2015) 4:29509. doi: 10.3402/jev.v4.29509

20. van der Pol E, Boing AN, Harrison P, Sturk A, Nieuwland R. Classification, functions, and clinical relevance of extracellular vesicles. Pharmacol Rev. (2012) 64:676-705. doi: 10.1124/pr.112.005983

21. Tang Y-T, Huang Y-Y, Zheng L, Qin S-H, Xu X-P, An T-X, et al. Comparison of isolation methods of exosomes and exosomal RNA from cell culture medium and serum. Int J Mol Med. (2017) 40:834-44. doi: 10.3892/ijmm.2017.3080

22. Nordin JZ, Lee Y, Vader P, Mäger I, Johansson HJ, Heusermann $\mathrm{W}$, et al. Ultrafiltration with size-exclusion liquid chromatography for high yield isolation of extracellular vesicles preserving intact biophysical and functional properties. Nanomedicine: Nanotechnol Biol Med. (2015) 11:879-83. doi: 10.1016/j.nano.2015.01.003

23. Helwa I, Cai J, Drewry MD, Zimmerman A, Dinkins MB, Khaled $\mathrm{ML}$, et al. A comparative study of serum exosome isolation using differential ultracentrifugation and three commercial reagents. PLoS ONE. (2017) 12:e0170628. doi: 10.1371/journal.pone.01 70628

24. Conforti A, Scarsella M, Starc N, Giorda E, Biagini S, Proia A, et al. Microvescicles derived from mesenchymal stromal cells are not as effective as their cellular counterpart in the ability to modulate immune responses in vitro. Stem Cells Dev. (2014) 23:2591-9. doi: 10.1089/scd. 2014.0091

Conflict of Interest Statement: The authors declare that the research was conducted in the absence of any commercial or financial relationships that could be construed as a potential conflict of interest.

Copyright $\odot 2019$ Carreras-Planella, Monguió-Tortajada, Borràs and Franquesa. This is an open-access article distributed under the terms of the Creative Commons Attribution License (CC BY). The use, distribution or reproduction in other forums is permitted, provided the original author(s) and the copyright owner(s) are credited and that the original publication in this journal is cited, in accordance with accepted academic practice. No use, distribution or reproduction is permitted which does not comply with these terms. 